\title{
Biosensor of stress hormone cortisol based on molecular imprinted polymer
}

\author{
Supannika Klangphukhiew $^{1}$, Roongnapa Srichana ${ }^{2}$, Peter A. Lieberzeit ${ }^{3}$, Rina Patramanon ${ }^{1,4}$ * \\ ${ }^{1}$ Department of Biochemistry, Faculty of Science, Khon Kaen University, Khon Kaen, 40002, Thailand \\ ${ }_{2}^{2}$ Molecular Recognition Materials Research Unit, Department of Pharmaceutical Chemistry, Faculty of \\ Pharmaceutical Sciences, Prince of Songkla University, Hat Yai, 90112, Thailand \\ ${ }^{3}$ Department of Physical Chemistry, Faculty of Chemistry, University of Vienna, Vienna, 1090, Austria \\ ${ }^{4}$ Protein and Proteomics Research Center for Commercial and Industrial Purposes (ProCCI), Khon \\ Kaen University, Khon Kaen, 40002, Thailand
}

narin@kku.ac.th

\begin{abstract}
Cortisol is one of the key hormones that is involved in stress response and an indicator of chronic psychological disorders. It is produced by zona fasciculata and can be found in blood serum and saliva. This study describes the fabrication of cortisol biosensor using molecular imprinted polymers (MIPs) as recognition unit. The $642.3 \pm 35.8 \mathrm{~nm}$-core shell cortisol-MIP was prepared by methacrylic acid and acrylamide with cortisol as a target molecule in a ratio of 2:1:1. Electron and atomic force microscope techniques were used to characterize the physical properties of cortisol-MIP. The binding characteristics were evaluated using covalent patterns changing in Fourier transform infrared (FTIR) spectra and massive response by quartz crystal microbalance (QCM) technique. Cortisol-MIP was integrated into the sensor and coated on the disposable screen-printed carbon electrode (SPCE) for portable electrochemical analysis, which displayed $1.28-20 \mathrm{mM}$ detection range and high selectivity compared to three other steroid analogs.
\end{abstract}

Key words: Molecular imprinted polymer (MIP), Stress hormone, Cortisol, Biosensor

\section{Introduction}

Chronic stress is an increasingly common disorder in modern life, caused by rising working hours and financial behaviors, and can lead to other diseases and accelerated signs of aging. Cortisol has been used as a well-known commercial stress biomarker. A homeostasis response to psychological stress is indicated by an increased level of cortisol, which is produced in the hypothalamus-pituitary-adrenal (HPA) axis. It can be found in blood serum, urine, saliva, sweat and hair, while it can be measured by ELISA and ECILA in hospitals [1].

In this study, we aim to fabricate portable cortisol biosensors using a stable receptor, namely MIPs. MIPs have been used to generate a mimicrecognition site by imprinting of unnatural polymers with a target molecule containing specific cavities on their surface. The selective detection of structures and superstructures is based on geometrical interactions which define both dimensions and proposed functional groups. Compared to traditional approaches, MIP-based techniques can achieve higher stability in a wide range of conditions, such as high temperatures or non-physiological $\mathrm{pH}$. Other advantages include low manufacturing cost and straightforward synthesis. Core-shell shaped polymers allow for a stable structure with a high number of cavities on the external surface [2]. MIP has been integrated with several types of transducers, especially electrochemical methods.

Herein, a small screen-printed carbon electrode was coated with core-shell MIP containing cortisol specific cavities, as shown in Figure 1. The changes of signal responses were detected by handheldelectrochemical devices in term of cyclic voltrametry suitable to develop small devices as miniature biosensors. 


\section{Material and methods}

Cortisol-MIP particles were obtained using the multistep swelling and polymerization protocol with cortisol as a target molecule. Styrene bead was used as the core shell. The microemulsion was reacted by 2,2' -azobisbutyronitrile (AIBN; Aldrich Chemie, Milwaukee, WI, USA) with polyvinylalcohol in toluene solvent. Poly methacrylic acid-co ethylene glycol dimethacrylate and N, N' -(1,2-Dihydroxyethylene)bis-acrylamide were polymerized with cortisol as template, stirring at $70^{\circ} \mathrm{C}$ under nitrogen stream for 24 hours. The cortisol template was washed with $10 \%$ acetic acid in methanol. The cortisol-MIP particles in suspension form were homogenized by High pressure homogenizer. The particle size was examined by Zetasizer. The physical properties of core shell MIP were investigated by SEM, TEM, AFM and XRD and correlated to a changing of binding properties by FTIR and QCM. After that, $4 \mu \mathrm{L}$ of Cortisol MIP suspension were dropped on screenprinted carbon electrode (SPCE) surface with 20\% solgel mixture and incubated at $65^{\circ} \mathrm{C}$ overnight to fix the cortisol MIP particles on SPCE surface. Electrochemical measurements were performed using a portable 910 PSTAT Mini (Metrohm autolab) connected with a computer system.

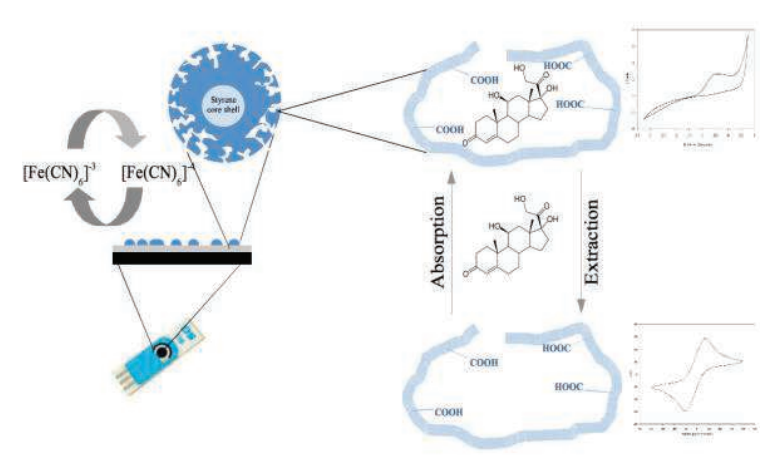

Figure 1. Schematic illustration of MIP particle modified screen-printed carbon electrode detecting cortisol.

\section{Results and discussion}

During the synthesis, the suitable ratio of methacrylic acid:acrylamide:cortisol, which allowed the formation of 642.3 $\pm 35.8-n m$ homogenous particles, was 2:1:1. The surface of MIP was rougher than non-imprinted polymer (NIP; the same reaction without the cortisol template). FTIR spectra show that cortisol-MIP particles could rebind other cortisol molecules. For measurement, the chromatogram was obtained by cyclic voltammetry (CV) analysis in $5 \times 10^{-3} \mathrm{~mol} / \mathrm{L}$ $\mathrm{K}_{3}\left[\mathrm{Fe}(\mathrm{CN})_{6}\right] / \mathrm{K}_{4}\left[\mathrm{Fe}(\mathrm{CN})_{6}\right]$ in $1 \times 10^{-1} \mathrm{~mol} / \mathrm{L} \mathrm{KCl}$. CV was scanned at different potential values from $0.45 \mathrm{~V}$ to $0.15 \mathrm{~V}$, at a $0.02 \mathrm{~V} / \mathrm{s}$ scan rate with 0.002 step of potential. The cyclic voltammograms of the SPCE modified with cortisol-MIP particles were indicated by the increase of redox peak at $0.2 \mathrm{~V}$. The detection range of cortisol concentrations was from 1.28 to $20 \mathrm{nM}$ with linear regression equation: $\Delta \mathrm{l}=1.92 \times 10^{-3}$ $\log \left[\mathrm{C}_{\text {cortisol }}\right]+13.72$ at a correlation coefficient of 0.9804 (Figure 2, 3).

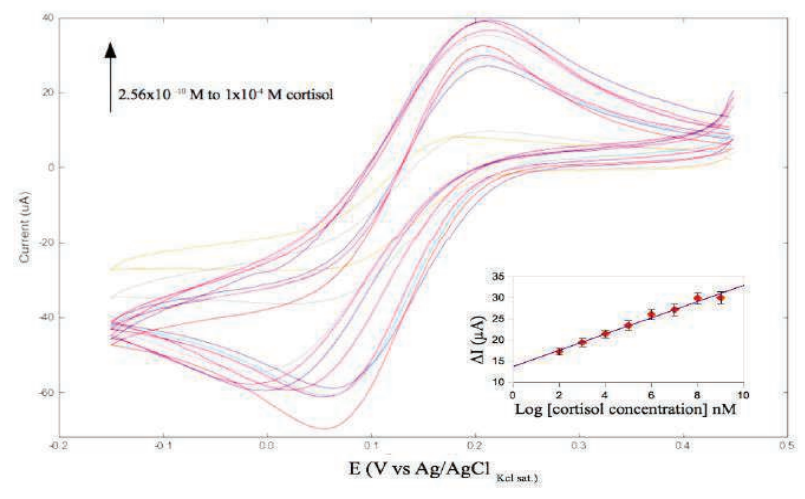

Figure 2. Cyclic voltammogram of MIP-modified SPCE responses to a wlde range of cortisol concentrations.

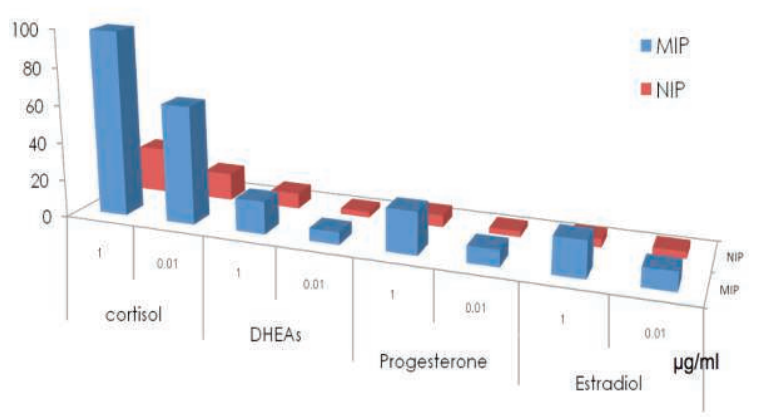

Figure 3. Percentage of MIP-modified SPCE responses to 0.01 and $1 \mu \mathrm{g} / \mathrm{ml}$ cortisol, dehydroepiandrosterone sulphate (DHEAs), progesterone and estradiol, respectively.

\section{Conclusions}

We reported the fabrication of cortisol stress sensor using shell MIPs as highly stable receptors combined with screen printed carbon electrodes and hand-held PSTAT as portable electrochemical device exhibiting a detection limit of $1.28 \mathrm{~nm}$ to rebind cortisol with high selectivity. This is a preventive healthcare attempt to develop biosensors for monitoring personal health in medical science for well-being.

\section{Acknowledgements}

This work has been funded by the Development and Promotion for Science and Technology talents project (DPST) by the Institute for the Promotion of Teaching Science and Technology. Part of the work has been supported by Synchrotron Light Research Institute funds 2017.

\section{References}

[1] Kaushik, A.; Vasudev, A.; Arya, S.K.; Pasha, S.K.; Bhansali, S. Recent advances in cortisol sensing technologies for point-of-care application. Biosens. Bioelectron. 2014, 53, 499-512, doi:10.1016/j.blos.2013.09.060.

[2] Wackerlig, J.; Lieberzeit, P.A. Molecularly imprinted polymer nanoparticles in chemical sensing-Synthesis, characterization and application. Sens. Actuators B Chem. 2015, 207, 144-157, doi:10.1016/j.snb.2014.09.094. 